\title{
An axiomatic theory of non-absolutely convergent integrals in $\mathbb{R}^{n}$
}

\author{
by
}

\author{
W. B. J u rkat and D. J. F. Nonnen macher (Ulm)
}

\begin{abstract}
We introduce an axiomatic approach to the theory of non-absolutely convergent integrals. The definition of our $\nu$-integral will be descriptive and depends mainly on characteristic null conditions. By specializing our concepts we will later obtain concrete theories of integration with natural properties and very general versions of the divergence theorem.
\end{abstract}

Introduction. Suppose that $F: \mathbb{R} \rightarrow \mathbb{R}$ is differentiable everywhere and that we seek an integration process which integrates $f=F^{\prime}$ always to the expected value. Since not all derivatives are absolutely integrable we need an extension of Lebesgue integration. Denjoy $(1912,1916,1917)$ was the first to solve this problem by a transfinite construction. Lusin $(1912,1916,1917)$ gave a much simpler descriptive definition by characterizing the associated interval function $F(b)-F(a)$. Directly constructive definitions in terms of Riemann sums (involving $f$ ) were given much later by Kurzweil (1957) and Henstock (1961). A partially constructive definition (involving major and minor (interval) functions) was given by Perron (1914).

Similar results for dimension $n>1$ seem much desirable. Here one would, e.g., consider an $n$-dimensional vector field $\vec{v}$ which is differentiable everywhere on $\mathbb{R}^{n}$ and the integral $\int_{A} \operatorname{div} \vec{v}$ over reasonable sets $A$, not just intervals. We look for an integration process which expresses this integral (without further assumptions) in terms of $\vec{v}$ on $\partial A$ in the expected way. Today there are essentially three lines of thought to achieve this goal.

In the papers by Jarník-Kurzweil [Jar-Ku 1-3], directly constructive definitions (in terms of Riemann sums) are given for a certain PU-integral (partition of unity). In dimension $n=2$ the sets $A$ considered are bounded by piecewise $C^{1}$ Jordan curves, while $A=\mathbb{R}^{n}$ for general $n$, with certain exceptional points allowed where differentiability is replaced by weaker conditions.

1991 Mathematics Subject Classification: 26A39, 26B30, 26B20, 28 A15. 
The papers by Pfeffer [Pf 1-3] and Pfeffer-Yang [Pf-Ya] begin with a partially constructive definition of a variational integral (which can also be characterized, cf. [Pf 3]) and then extend it by transfinite induction. Pfeffer treats sets $A \in B V$ (for instance compact sets with $|\partial A|_{n-1}<\infty$, cf. [Ju]) and allows general $(n-1)$-dimensional exceptional sets, where $\vec{v}$ is only continuous or bounded.

Finally, Jurkat [Ju] uses Riemann sums restricted by various control conditions. Here the basic tool is a decomposition theorem and the resulting divergence theorem is essentially the same as Pfeffer's. The method of control conditions is very flexible and was extended by Nonnenmacher [No] now allowing further exceptional points in the divergence theorem, where $\vec{v}$ satisfies only Lipschitz conditions of non-negative orders.

In the meantime we have extended the method still further so that now also Lipschitz conditions of negative orders $>1-n$ can be treated. In fact, based on Jurkat's decomposition theorem, there is a great variety of integrals which could be used and lead to "concrete" theories. So it was natural to look for common underlying ideas, and this led to the axiomatic theory which we present in this paper. It will be useful to explain some of the general ideas:

A constructive definition associates with a point function $f$ a number, its integral $\int f$, while a descriptive definition associates with $f$ an additive set function $F$ whose derivative $\dot{F}$ (e.g., the ordinary derivative in the sense of [Saks]) equals $f$ almost everywhere. Here one needs further restrictions on $F$ such that $f=0$ implies $F=0$ (uniqueness). Ideally, we look for directly constructive definitions (with Riemann sums) which are equivalent to such a descriptive definition. In our concrete theories this equivalence can be established, and we found that certain null conditions, formulated in terms of control conditions, express the relevant properties of $F$.

This occurred first in Jurkat-Knizia [Ju-Kn], also in Nonnenmacher [No], and again in Pfeffer [Pf 3].

Another problem with non-absolutely convergent integrals is the additivity, which is lacking for instance in Mawhin [Maw], Jurkat-Knizia [Ju-Kn], and also for Pfeffer's variational integral [Pf 2, Prop. 5.7 and Ex. 5.21], which is only partially additive. The following general principle may help. Suppose we have a concept of integrability and integral for functions $f: \mathbb{R}^{n} \rightarrow \mathbb{R}$, which is linear and such that $f \chi_{B}$ is also integrable for a certain class $\mathcal{B}$ of sets $B\left(\chi_{B}\right.$ being the characteristic function of $\left.B\right)$. Then we can define $\int_{A} f$ by $\int f \chi_{A}$ provided that $f \chi_{A}$ is integrable ( $A$ arbitrary). If $A$ is the disjoint union of $B_{1}$ and $B_{2}$ in $\mathcal{B}$ we see from $f \chi_{A}=f \chi_{B_{1}}+f \chi_{B_{2}}\left(f \chi_{B_{i}}=f \chi_{A} \cdot \chi_{B_{i}}\right)$ that $f$ is integrable over $A$ iff $f$ is integrable over $B_{1}$ and $B_{2}$; moreover, in that case

$$
\int_{A} f=\int_{B_{1}} f+\int_{B_{2}} f
$$


Our present paper is organized as follows. In Section 0 we explain that our abstract process of integration depends on the concepts of "reasonable" sets, of differentiability, and of control conditions. The fundamental definitions (and axioms) are given in Sections 1-3. The relevant properties of our $\nu$-integral ( $\nu$ refers to the null conditions) are proved in Sections 4 and 5 (e.g., it is an extension of the Lebesgue integral). In Section 6 we give our version of the Saks-Henstock lemma which will be used in our concrete theories to show the equivalence of the constructive and descriptive definitions. We also have a transformation formula based on an additional axiom (Section 7). Specializing our concepts and results we obtain mainly two concrete theories, which will be published later.

0. Preliminaries. $\mathbb{N}, \mathbb{R}$ and $\mathbb{R}^{+}$are the sets of all positive integers, all real numbers and all positive real numbers, respectively, and $n \in \mathbb{N}$ is assumed to be fixed throughout the paper. In $\mathbb{R}^{n}$ we work with the usual inner product $x \cdot y=\sum_{i=1}^{n} x_{i} y_{i}$ and the associated norm $\|\cdot\|$. If $A \subseteq \mathbb{R}^{n}$ and $x \in \mathbb{R}^{n}$, then $A^{\circ}, \bar{A}, \partial A, d(A)$ and $\operatorname{dist}(x, A)$ denote respectively the interior, closure, boundary, diameter of $A$ and the distance from the point $x$ to the set $A$. For $x \in \mathbb{R}^{n}$ and $r>0$ let $B(x, r)=\left\{y \in \mathbb{R}^{n}:\|y-x\| \leq r\right\}$. $\mathcal{P}\left(\mathbb{R}^{n}\right)$ denotes the power set of $\mathbb{R}^{n}$; the complement of $A \subset \mathbb{R}^{n}$, i.e. the set $\mathbb{R}^{n}-A$, is sometimes denoted by $A^{\mathrm{c}}$.

In $\mathbb{R}^{n}$ we use the $n$-dimensional outer Lebesgue measure $|\cdot|_{n}$, and a subset $E$ of $\mathbb{R}^{n}$ with $|E|_{n}=0$ is called an $n$-null set or just a null set. Terms like measurable and almost everywhere (a.e.) always refer to the measure $|\cdot|_{n}$.

Throughout, an interval is assumed to be compact and non-degenerate.

Now we like to explain in general terms the underlying structure we need to define our process of integration. Our descriptive integral will be an additive set function defined on a class $\mathcal{B}$ of sets. This function shall be differentiable a.e.; so we need a differentiation class, that is, a system of sets from $\mathcal{B}$ which is used in forming the set derivative. Thinking of Vitali regularity, which is controlled by a parameter $K>0$, our differentiation class will also depend upon $K$ and will be denoted by $\mathcal{D}(K)$. Most important will be the formulation of our null conditions which require

$$
\sum\left|F\left(A_{k}\right)\right| \leq \varepsilon \quad \text { (small) }
$$

provided that the sets $A_{k} \in \mathcal{B}$ are suitably restricted (depending on $\varepsilon>0$ ). These restrictions are formulated in terms of control conditions. We could require, for instance (as in $\mathrm{AC}$ ), that

$$
\left|\bigcup A_{k}\right|_{n} \leq \Delta \quad \text { (small) }
$$

with $\Delta=\Delta(\varepsilon)>0$ or that certain regularity parameters $K>0$ be bounded. 
In general, our control conditions will depend on two parameters, $K$ and $\Delta$, and define (purely geometrically) the finite sequences $\left\{A_{k}\right\}$ which are admitted. Thus with a control condition $C$ there will be associated the classes $C(K, \Delta)$ of such sequences $\left\{A_{k}\right\}$, and each control condition will define a corresponding null condition. In order to prove uniqueness, i.e. $F(A)=0$ for $A \in \mathcal{B}$, we will decompose $A$ into finitely many parts $A_{k}$. Some of them will make a small contribution because $\dot{F}$ is zero there, and others because of a corresponding null condition. So it will be important that there exist decompositions where the parts can be grouped so that they satisfy our control conditions, and we will use two types of control conditions (see our decomposition axiom). We will have a system $\dot{\Gamma}$ of control conditions which we use on the "regular" parts of the decomposition and another system $\Gamma$ for the remaining parts. All resulting null conditions will represent a weakening of AC.

In total we will have a quadruple $\nu=(\mathcal{B}, \mathcal{D}, \dot{\Gamma}, \Gamma)$, and our process of integration will only depend on $\nu$.

1. Semi-rings, differentiation sets and control conditions. In the following we will give some basic definitions and our assumptions on $\mathcal{B}, \mathcal{D}$, $\dot{\Gamma}$ and $\Gamma$.

We suppose that $\mathcal{B}$ is a system of subsets of $\mathbb{R}^{n}$ containing all intervals and such that

(i) $B$ is compact and $|\partial B|_{n}=0$ for all $B \in \mathcal{B}$.

(ii) If $A, B \in \mathcal{B}$ then $A \cap B \in \mathcal{B}$ and $A-B^{\circ}=\bigcup A_{k}$, a finite union with all $A_{k} \in \mathcal{B}$ having disjoint interiors.

We call $\mathcal{B}$ a semi-ring (cf. [Weir, p. 86]).

For each $K>0$ we have a differentiation class $\mathcal{D}(K) \subseteq \mathcal{B}$ satisfying $d(B)^{n} \leq K|B|_{n}$ for any $B \in \mathcal{D}(K)$, and we suppose that $\mathcal{D}\left(K_{1}\right) \subseteq \mathcal{D}\left(K_{2}\right)$ whenever $K_{1} \leq K_{2}$. Furthermore, we assume the existence of an absolute constant $K_{*}=K_{*}(n)>0$ with the property that for all $K \geq K_{*}$ any interval $I$ with $d(I)^{n} \leq K|I|_{n}$ belongs to $\mathcal{D}(K)$.

$\mathcal{D}$ associates with each positive $K$ the differentiation class $\mathcal{D}(K)$.

A control condition $C$ associates with any positive parameters $K$ and $\Delta$ a class $C(K, \Delta)$ of finite sequences $\left\{A_{k}\right\}$ with $A_{k} \in \mathcal{B}$ and has the following properties:

(i) $C(K, \Delta)$ is increasing in $K$ and $\Delta$, i.e. if $K_{1} \leq K_{2}$ and $\Delta_{1} \leq \Delta_{2}$ then $C\left(K_{1}, \Delta_{1}\right) \subseteq C\left(K_{2}, \Delta_{2}\right)$.

(ii) If $\left\{A_{k}\right\} \in C(K, \Delta)$ then every subsequence of $\left\{A_{k}\right\}$ also belongs to $C(K, \Delta)$.

Furthermore, with $C$ there is associated a system $\mathcal{E}(C)$ of subsets of $\mathbb{R}^{n}$ such that $E \in \mathcal{E}(C)$ implies $F \in \mathcal{E}(C)$ for any $F \subseteq E$. 
Let $E \subseteq \mathbb{R}^{n}$ and $\delta: E \rightarrow \mathbb{R}^{+}$be given. Then a finite sequence of pairs $\left\{\left(x_{k}, A_{k}\right)\right\}$ with $x_{k} \in A_{k} \in \mathcal{B}, A_{i}^{\circ} \cap A_{j}^{\circ}=\emptyset(i \neq j), x_{k} \in E$ and $d\left(A_{k}\right)<\delta\left(x_{k}\right)$ is called $(E, \delta)$-fine. If in addition $E=\bigcup A_{k}$ we call $\left\{\left(x_{k}, A_{k}\right)\right\}$ a $\delta$-fine partition of $E$. [Later on the property of being $(E, \delta)$-fine will often be combined with some control condition $C$, where $E \in \mathcal{E}(C)$.]

Remark 1.1. Let $E \subseteq \mathbb{R}^{n}$ be a null set and let $f: E \rightarrow \mathbb{R}$. Then for any $\varepsilon>0$ there exists a $\delta: E \rightarrow \mathbb{R}^{+}$such that $\sum\left|f\left(x_{k}\right)\right|\left|A_{k}\right|_{n} \leq \varepsilon$ for any $(E, \delta)$-fine sequence $\left\{\left(x_{k}, A_{k}\right)\right\}$.

Indeed, fix $\varepsilon>0$, let $E=\bigcup_{j \in \mathbb{N}} E_{j}$ with $E_{j}=\{x \in E: j-1 \leq|f(x)|<j\}$ and find open sets $G_{j} \supseteq E_{j}$ with $\left|G_{j}\right|_{n} \leq \varepsilon /\left(j 2^{j}\right)$. For $x \in E_{j}$ choose a $\delta(x)>0$ such that $B(x, \delta(x)) \subseteq G_{j}$, and let $\left\{\left(x_{k}, A_{k}\right)\right\}$ be an $(E, \delta)$-fine sequence. Then

$$
\sum\left|f\left(x_{k}\right)\right|\left|A_{k}\right|_{n} \leq \sum_{j \in \mathbb{N}} \sum_{x_{k} \in E_{j}} j\left|A_{k}\right|_{n} \leq \sum_{j \in \mathbb{N}} j\left|\bigcup_{x_{k} \in E_{j}} A_{k}\right|_{n} \leq \sum_{j \in \mathbb{N}} j\left|G_{j}\right|_{n} \leq \varepsilon
$$

as desired.

Here we used the fact that $\left|\bigcup_{i=1}^{m} A_{i}\right|_{n}=\sum_{i=1}^{m}\left|A_{i}\right|_{n}$ for any $A_{1}, \ldots, A_{m}$ $\in \mathcal{B}$ having disjoint interiors, since $\left|\partial A_{i}\right|_{n}=0$ for all $i$.

Comparison of control conditions. Let $C_{1}$ and $C_{2}$ be two control conditions. We call $C_{1}$ stronger than $C_{2}\left(C_{1} \succeq C_{2}\right)$ if $\forall K_{1}>0 \exists K_{2}>0 \forall \Delta_{2}>0$ $\exists \Delta_{1}>0 \exists \delta: \mathbb{R}^{n} \rightarrow \mathbb{R}^{+}$such that for any $\left(\mathbb{R}^{n}, \delta\right)$-fine sequence $\left\{\left(x_{k}, A_{k}\right)\right\}$, if $\left\{A_{k}\right\} \in C_{1}\left(K_{1}, \Delta_{1}\right)$ then $\left\{A_{k}\right\} \in C_{2}\left(K_{2}, \Delta_{2}\right)$.

Remark 1.2. An easy check shows that the relation $\succeq$ is reflexive and transitive.

A system $\mathcal{C}$ of control conditions is said to be ordered if for any $C_{1}$ and $C_{2}$ in $\mathcal{C}$, either $C_{1} \succeq C_{2}$ or $C_{2} \succeq C_{1}$.

A control condition $C^{*}$ is said to be a minimal element of an ordered system $\mathcal{C}$ of control conditions if $C^{*} \in \mathcal{C}$ and $C \succeq C^{*}$ for any $C \in \mathcal{C}$.

We suppose $\dot{\Gamma}$ to be an ordered system of control conditions having the following properties:

$\left(\dot{\Gamma}_{1}\right) \quad$ For any $C \in \dot{\Gamma}, K>0, \Delta>0$ and each $\left\{A_{k}\right\} \in C(K, \Delta)$ we have $A_{k} \in \mathcal{D}(K)$ for all $k$.

$\left(\dot{\Gamma}_{2}\right) \quad$ For every null set $E \subseteq \mathbb{R}^{n}$ there is an element $C \in \dot{\Gamma}$ with $E \in \mathcal{E}(C)$.

Finally, we assume $\Gamma$ to be an ordered system of control conditions which is disjoint from $\dot{\Gamma}$ and contains a minimal element $C^{*}$ which satisfies the following conditions:

$\left(\Gamma_{1}\right) \quad \partial A \in \mathcal{E}\left(C^{*}\right)$ for all $A \in \mathcal{B}$.

$\left(\Gamma_{2}\right) \quad$ For any $\varepsilon>0$ and $K>0$ there are $\Delta>0$ and $\delta: \mathbb{R}^{n} \rightarrow \mathbb{R}^{+}$such that $\left|\bigcup A_{k}\right|_{n}<\varepsilon$ for every $\left(\mathbb{R}^{n}, \delta\right)$-fine sequence $\left\{\left(x_{k}, A_{k}\right)\right\}$ with $\left\{A_{k}\right\} \in C^{*}(K, \Delta)$. 
Motivation. Condition $\left(\Gamma_{1}\right)$ will be important when we restrict our integral to sets $A \in \mathcal{B}$ (see Thm. II in Section 4) while by $\left(\Gamma_{2}\right)$ the null condition corresponding to $C^{*}$ (see Section 3 ) will be a weakening of the classical absolute continuity. This in connection with $\left(\dot{\Gamma}_{2}\right)$ will be important to integrate all Lebesgue integrable functions.

2. The axioms of decomposition and intersection and the decomposition lemma. In order to guarantee satisfactory properties of the integration process which will be induced by our quadruple $\nu=(\mathcal{B}, \mathcal{D}, \dot{\Gamma}, \Gamma)$ we assume two further axioms.

A division of $\mathbb{R}^{n}$ consists of a set $\dot{E}$ and a sequence of pairs $\left(E_{i}, C_{i}\right)_{i \in \mathbb{N}}$, where $C_{i} \in \Gamma \cup \dot{\Gamma}$ and the $E_{i}$ are null sets in $\mathcal{E}\left(C_{i}\right)$ such that $\mathbb{R}^{n}$ is the disjoint union of all the $E_{i}$ and $\dot{E}$.

Analogously for $A \in \mathcal{B}$ we call $\dot{E},\left(E_{i}, C_{i}\right)_{i \in \mathbb{N}}$ a division of $A$ if $C_{i} \in \Gamma \cup \dot{\Gamma}$, $E_{i} \in \mathcal{E}\left(C_{i}\right), \dot{E} \subseteq A^{\circ}$ with $|A-\dot{E}|_{n}=0$ and if $A$ is the disjoint union of all the $E_{i}$ and $\dot{E}$.

We first formulate the decomposition axiom which guarantees for each interval $I$ the existence of suitable partitions corresponding to arbitrary divisions of the interval. In concrete situations the verification of this axiom will be the crucial point.

AxIom 1 (decomposition). Let $I$ be any interval in $\mathbb{R}^{n}$ and $\dot{E},\left(E_{i}, C_{i}\right)_{i \in \mathbb{N}}$ be any division of $I$. Then there shall exist positive numbers $K^{*}$ and $K_{i}^{*}$ such that for any $\Delta_{i}>0$ and $\delta: I \rightarrow \mathbb{R}^{+}$there is a $\delta$-fine partition $\left\{\left(x_{k}, A_{k}\right)\right\}$ of $I$ with the following properties:

- if $x_{k} \in \dot{E}$ then $A_{k}$ belongs to $\mathcal{D}\left(K^{*}\right)$,

- $\left\{A_{k}: x_{k} \in E_{i}\right\} \in C_{i}\left(K_{i}^{*}, \Delta_{i}\right)$ for all $i \in \mathbb{N}$.

Axiom 2 will be important when restricting the integral to $A \in \mathcal{B}$; it essentially states that if $\left\{A_{k}\right\}$ belongs to a control condition of $\Gamma$ then $\left\{A \cap A_{k}\right\}$ belongs to the same control condition.

Axiom 2 (intersection). For any $C \in \Gamma, E \in \mathcal{E}(C)$ and any $A \in \mathcal{B}$ the following shall be true: $\forall K_{1}>0 \exists K_{2}>0 \forall \Delta_{2}>0 \exists \Delta_{1}>0 \exists \delta: E \cap A \rightarrow \mathbb{R}^{+}$ such that $\left\{A \cap A_{k}\right\} \in C\left(K_{2}, \Delta_{2}\right)$ for any $(E \cap A, \delta)$-fine sequence $\left\{\left(x_{k}, A_{k}\right)\right\}$ with $\left\{A_{k}\right\} \in C\left(K_{1}, \Delta_{1}\right)$.

Both axioms together imply that each $A \in \mathcal{B}$ can be partitioned in a certain way.

Decomposition Lemma. Let $A \in \mathcal{B}$ and $\dot{E},\left(E_{i}, C_{i}\right)_{i \in \mathbb{N}}$ be a division of $A$. Then there exist positive numbers $K^{*}$ and $K_{i}^{*}$ such that for any $\Delta_{i}>0$ and $\delta: A \rightarrow \mathbb{R}^{+}$there is a $\delta$-fine partition $\left\{\left(x_{k}, A_{k}\right)\right\}$ of $A$ with the following properties: 
(i) - if $x_{k} \in \dot{E}$ then $A_{k} \in \mathcal{D}\left(K^{*}\right)$,

- $\left\{A_{k}: x_{k} \in E_{i}\right\} \in C_{i}\left(K_{i}^{*}, \Delta_{i}\right)$ if $C_{i} \in \Gamma$,

- $\left\{A_{k}: x_{k} \in E_{i} \cap A^{\circ}\right\} \in C_{i}\left(K_{i}^{*}, \Delta_{i}\right)$ if $C_{i} \in \dot{\Gamma}$,

(ii) $\left\{A_{k}: x_{k} \in E_{i} \cap \partial A\right\} \in C^{*}\left(K_{i}^{*}, \Delta_{i}\right)$ if $C_{i} \in \dot{\Gamma}$.

Proof. Choose an interval $I$ with $A \subseteq I^{\circ}$. Then

$\dot{E} \cup\left(I^{\circ}-A\right),\left(\partial I, C^{*}\right),\left(E_{i} \cap A^{\circ}, C_{i}\right)_{C_{i} \in \dot{\Gamma}},\left(E_{i} \cap \partial A, C^{*}\right)_{C_{i} \in \dot{\Gamma}},\left(E_{i}, C_{i}\right)_{C_{i} \in \Gamma}$

is a division of $I$ and thus Axiom 1 yields positive numbers $K^{*},{ }^{1} K_{i}^{*}\left(C_{i} \in \dot{\Gamma}\right)$, ${ }^{2} K_{i}^{*}\left(C_{i} \in \dot{\Gamma}\right)$ and ${ }^{2} K_{i}^{*}\left(C_{i} \in \Gamma\right)$.

The pair $\left(\partial I, C^{*}\right)$ will not be needed in the following and before we complete the application of Axiom 1 we use Axiom 2 to determine the relevant $\Delta$ 's and $\delta$.

For ${ }^{2} K_{i}^{*}$ choose $\widetilde{K}_{i}^{*}>0$ by Axiom 2 , set

$$
K_{i}^{*}= \begin{cases}\max \left({ }^{1} K_{i}^{*}, \widetilde{K}_{i}^{*}\right) & \text { if } C_{i} \in \dot{\Gamma}, \\ \widetilde{K}_{i}^{*} & \text { if } C_{i} \in \Gamma,\end{cases}
$$

and let $\Delta_{i}>0$ and $\delta: A \rightarrow \mathbb{R}^{+}$be given. Extend $\delta$ to $I$ by $\delta(x)=\operatorname{dist}(x, A)$ for $x \in I-A$ and assume (without loss of generality) $\delta(x)<\operatorname{dist}\left(x, \mathbb{R}^{n}-A^{\circ}\right.$ ) if $x \in A^{\circ}$.

For $\Delta_{i}>0$ choose, again by Axiom 2, $\widetilde{\Delta}_{i}>0$ and $\delta_{i}: E_{i} \cap \partial A \rightarrow \mathbb{R}^{+}$ or $\delta_{i}: E_{i} \rightarrow \mathbb{R}^{+}$depending on whether $C_{i} \in \dot{\Gamma}$ or $C_{i} \in \Gamma$. Assuming $\delta \leq \delta_{i}\left(i \in \mathbb{N}\right.$ ) we can find by Axiom 1 (using $\Delta_{i}$ and $\left.\widetilde{\Delta}_{i}\right)$ a $\delta$-fine partition $\left\{\left(x_{k}, I_{k}\right)\right\}$ of $I$ with the following properties: if $x_{k} \in \dot{E}$ then $I_{k} \in \mathcal{D}\left(K^{*}\right)$; $\left\{I_{k}: x_{k} \in E_{i} \cap A^{\circ}\right\} \in C_{i}\left({ }^{1} K_{i}^{*}, \Delta_{i}\right)$ if $C_{i} \in \dot{\Gamma} ;\left\{I_{k}: x_{k} \in E_{i} \cap \partial A\right\} \in$ $C^{*}\left({ }^{2} K_{i}^{*}, \widetilde{\Delta}_{i}\right)$ if $C_{i} \in \dot{\Gamma}$; and $\left\{I_{k}: x_{k} \in E_{i}\right\} \in C_{i}\left({ }^{2} K_{i}^{*}, \widetilde{\Delta}_{i}\right)$ if $C_{i} \in \Gamma$.

Now set $A_{k}=A \cap I_{k}$ if $x_{k} \in A$. Then $\left\{\left(x_{k}, A_{k}\right)\right\}$ is a $\delta$-fine partition of $A$. Furthermore:

- if $x_{k} \in \dot{E}\left(\subseteq A^{\circ}\right)$ then $A_{k}=I_{k} \in \mathcal{D}\left(K^{*}\right)$,

- $\left\{A_{k}: x_{k} \in E_{i}\right\} \in C_{i}\left(\widetilde{K}_{i}^{*}, \Delta_{i}\right)$ for $C_{i} \in \Gamma$ by Axiom 2 since $\left\{\left(x_{k}, I_{k}\right)\right.$ : $\left.x_{k} \in E_{i}\right\}$ is an $\left(E_{i}, \delta_{i}\right)$-fine sequence with $\left\{I_{k}\right\} \in C_{i}\left({ }^{2} K_{i}^{*}, \widetilde{\Delta}_{i}\right)$, and

- analogously $\left\{A_{k}: x_{k} \in E_{i} \cap \partial A\right\} \in C^{*}\left(\widetilde{K}_{i}^{*}, \Delta_{i}\right)$ and $\left\{A_{k}: x_{k} \in\right.$ $\left.E_{i} \cap A^{\circ}\right\}=\left\{I_{k}: x_{k} \in E_{i} \cap A^{\circ}\right\} \in C_{i}\left({ }^{1} K_{i}^{*}, \Delta_{i}\right)$ for $C_{i} \in \dot{\Gamma}$.

Since all control conditions are non-decreasing in $K$ the proof is complete.

R e m ark 2.1. If the control condition $C^{*}$ only depends on $K$ we may replace (ii) of the lemma above by the condition $\left\{A_{k}: x_{k} \in \partial A \cap \bigcup_{C_{i} \in \dot{\Gamma}} E_{i}\right\} \in$ $C^{*}\left(K^{*}\right)$. In the proof one only has to replace the sequence $\left(E_{i} \cap \partial A, C^{*}\right)_{C_{i} \in \dot{\Gamma}}$ by the pair $\left(\partial A \cap \bigcup_{C_{i} \in \dot{\Gamma}} E_{i}, C^{*}\right)$. 
3. Set functions, null conditions and the $\nu$-integral. By a set function we mean a function $F: \mathcal{B} \rightarrow \mathbb{R}$.

- We call $F$ additive if $F(A)=\sum F\left(A_{k}\right)$ for any $A \in \mathcal{B}$ and every finite sequence $\left\{A_{k}\right\}$ with $A_{k} \in \mathcal{B}$ having disjoint interiors and $A=\bigcup A_{k}$. (It follows that $F(B)=0$ if $|B|_{n}=0$.)

- $F$ is said to have compact support if there exists an interval $I$ with $F(B)=0$ for every $B \in \mathcal{B}$ with $B \subseteq \mathbb{R}^{n}-I^{\circ}$. Any such $I$ is called an interval of support.

- $F$ is called differentiable at $x \in \mathbb{R}^{n}$ if there exists a real number $\alpha$ such that for any $\varepsilon>0$ and $K>0$ there is a $\delta=\delta(x)>0$ with

$$
\left.\left.|F(B)-\alpha| B\right|_{n}|\leq \varepsilon| B\right|_{n}
$$

for every $B \in \mathcal{D}(K)$ satisfying $x \in B$ and $d(B)<\delta$. In this case $\alpha$ is uniquely determined and denoted by $\dot{F}(x)$.

Re mark 3.1. Let $F: \mathcal{B} \rightarrow \mathbb{R}$ be additive with compact support and let $I$ and $\widetilde{I}$ be two intervals of support. Then we can write $I-\widetilde{I}^{\circ}=\bigcup A_{k}$ (a finite union) with $A_{k} \in \mathcal{B}$ having disjoint interiors and because $I=(I \cap \widetilde{I}) \cup \bigcup A_{k}$ it follows by additivity that $F(I)=F(I \cap \widetilde{I})+\sum F\left(A_{k}\right)=F(I \cap \widetilde{I})$ since all $A_{k} \subset \mathbb{R}^{n}-\widetilde{I}^{\circ}$. Analogously we get $F(\widetilde{I})=F(I \cap \widetilde{I})$, giving $F(I)=F(\widetilde{I})$.

In what follows, this unique number will be denoted by $F\left(\mathbb{R}^{n}\right)$.

Null conditions. Let $F: \mathcal{B} \rightarrow \mathbb{R}$ be a set function, $C$ a control condition, and $E \subseteq \mathbb{R}^{n}$. Then we say that $F$ satisfies the null condition corresponding to $C$ on $E$ (for short, $F$ satisfies $\mathcal{N}(C, E)$ ) if $\forall \varepsilon>0, K>0 \exists \Delta>0 \exists \delta$ : $E \rightarrow \mathbb{R}^{+}$such that $\sum\left|F\left(A_{k}\right)\right| \leq \varepsilon$ for any $(E, \delta)$-fine sequence $\left\{\left(x_{k}, A_{k}\right)\right\}$ with $\left\{A_{k}\right\} \in C(K, \Delta)$.

Re mark 3.2. (i) Let $E_{i}(i=1, \ldots, m)$ be disjoint sets, and $E=\bigcup_{i=1}^{m} E_{i}$. Then $F$ satisfies $\mathcal{N}\left(C, E_{i}\right)$ for all $i$ iff $F$ satisfies $\mathcal{N}(C, E)$.

For, if $F$ satisfies $\mathcal{N}\left(C, E_{i}\right)$ for all $i$ and if $\varepsilon, K>0$ then we can find $\Delta_{i}>0$ and $\delta_{i}: E_{i} \rightarrow \mathbb{R}^{+}$such that $\sum\left|F\left(A_{k}\right)\right| \leq \varepsilon / m$ for any $\left(E_{i}, \delta_{i}\right)$-fine sequence $\left\{\left(x_{k}, A_{k}\right)\right\}$ with $\left\{A_{k}\right\} \in C\left(K, \Delta_{i}\right)$. Set $\Delta=\min _{1 \leq i \leq m} \Delta_{i}$ and $\delta(x)=\delta_{i}(x)$ if $x \in E_{i}$ and let $\left\{\left(x_{k}, A_{k}\right)\right\}$ be an $(E, \delta)$-fine sequence with $\left\{A_{k}\right\} \in C(K, \Delta)$. Then

$$
\sum\left|F\left(A_{k}\right)\right|=\sum_{i} \sum_{x_{k} \in E_{i}}\left|F\left(A_{k}\right)\right| \leq \sum_{i} \varepsilon / m=\varepsilon .
$$

The other direction is obvious.

For countably many $E_{i}$ the result still holds provided $C$ does not depend on $\Delta$.

(ii) If $F$ satisfies $\mathcal{N}(C, E)$, then $F$ satisfies $\mathcal{N}\left(C^{\prime}, E\right)$ for any $C^{\prime} \succeq C$. Indeed, let $\varepsilon, K^{\prime}>0$. Take a $K>0$ witnessing $C^{\prime} \succeq C$. Then for $\varepsilon, K$ 
choose $\Delta>0$ and $\delta: E \rightarrow \mathbb{R}^{+}$witnessing that $F$ satisfies $\mathcal{N}(C, E)$ and take $\Delta^{\prime}>0$ and $\delta^{\prime}: \mathbb{R}^{n} \rightarrow \mathbb{R}^{+}$again using $C^{\prime} \succeq C$. Assume $\delta^{\prime} \leq \delta$ on $E$ and let $\left\{\left(x_{k}, A_{k}\right)\right\}$ be an $\left(E, \delta^{\prime}\right)$-fine sequence with $\left\{A_{k}\right\} \in C^{\prime}\left(K^{\prime}, \Delta^{\prime}\right)$. Then $\left\{A_{k}\right\} \in C(K, \Delta)$ and therefore $\sum\left|F\left(A_{k}\right)\right| \leq \varepsilon$.

(iii) Let $E \subseteq \mathbb{R}^{n}$ be a null set and $F$ be differentiable on $E$. Then for any $\varepsilon, K>0$ there exists a $\delta: E \rightarrow \mathbb{R}^{+}$such that $\sum\left|F\left(A_{k}\right)\right| \leq \varepsilon$ for any $(E, \delta)$-fine sequence $\left\{\left(x_{k}, A_{k}\right)\right\}$ with $A_{k} \in \mathcal{D}(K)$ for all $k$. In particular, $F$ satisfies $\mathcal{N}(C, E)$ for every $C \in \dot{\Gamma}$.

Proof. Choose an open set $G \supseteq E$ with $|G|_{n} \leq 1$ and let $\varepsilon, K>0$. Then for every $x \in E$ there is a $\delta(x)>0$ such that $B(x, \delta(x)) \subseteq G$ and $\left.\left.|F(B)-\dot{F}(x)| B\right|_{n}|\leq \varepsilon| B\right|_{n} / 2$ for any $B \in \mathcal{D}(K)$ with $x \in B$ and $d(B)<$ $\delta(x)$. Furthermore, by Remark 1.1 (making $\delta$ smaller if necessary), we may assume $\sum\left|\dot{F}\left(x_{k}\right)\right|\left|A_{k}\right|_{n} \leq \varepsilon / 2$ for any $(E, \delta)$-fine sequence $\left\{\left(x_{k}, A_{k}\right)\right\}$. Thus given an $(E, \delta)$-fine sequence $\left\{\left(x_{k}, A_{k}\right)\right\}$ with $A_{k} \in \mathcal{D}(K)$ for all $k$ we get

$$
\begin{aligned}
\sum\left|F\left(A_{k}\right)\right|-\sum\left|\dot{F}\left(x_{k}\right)\right|\left|A_{k}\right|_{n} & \leq\left.\sum\left|F\left(A_{k}\right)-\dot{F}\left(x_{k}\right)\right| A_{k}\right|_{n} \mid \\
& \leq \frac{\varepsilon}{2} \sum\left|A_{k}\right|_{n} \leq \frac{\varepsilon}{2}|G|_{n} \leq \frac{\varepsilon}{2}
\end{aligned}
$$

and therefore $\sum\left|F\left(A_{k}\right)\right| \leq \varepsilon$.

(iv) If $G: \mathcal{B} \rightarrow \mathbb{R}$ is another set function and if $c_{1}$ and $c_{2}$ are real numbers then $c_{1} F+c_{2} G$ satisfies $\mathcal{N}(C, E)$ provided that both $F$ and $G$ satisfy $\mathcal{N}(C, E)$.

An additive set function $F: \mathcal{B} \rightarrow \mathbb{R}$ is called a $\nu$-integral if the following two conditions are satisfied:

(1) There exists a division $\dot{E},\left(E_{i}, C_{i}\right)_{i \in \mathbb{N}}$ of $\mathbb{R}^{n}$ such that $F$ is differentiable on $\dot{E}$ and satisfies $\mathcal{N}\left(C_{i}, E_{i}\right)$ for all $i \in \mathbb{N}, \mathcal{N}\left(C^{*}, \dot{E}\right)$, and $\mathcal{N}\left(C^{*}, E_{i}\right)$ if $C_{i} \in \dot{\Gamma}$.

(2) There is an interval $I$ with $\dot{F}=0$ a.e. on $I^{\text {c }}$.

(Here (2) reflects the fact that later on we will integrate functions $f$ : $\mathbb{R}^{n} \rightarrow \mathbb{R}$ having compact support.)

Let $A \in \mathcal{B}, \mathcal{B}(A)=\{B \in \mathcal{B}: B \subseteq A\}, F: \mathcal{B}(A) \rightarrow \mathbb{R}$ (called a set function on $A), x \in A^{\circ}, C$ a control condition and $E \subseteq A$. Then it is clear what it means that $F$ is an additive set function on $\mathcal{B}(A)$ or that $F$ is differentiable at $x$ or that $F$ satisfies $\mathcal{N}(C, E)$ (just require all sets occurring in the definitions given above to be in $\mathcal{B}(A))$.

We call an additive set function $F: \mathcal{B}(A) \rightarrow \mathbb{R}$ a $\nu$-integral on $A$ if there exists a division $\dot{E},\left(E_{i}, C_{i}\right)_{i \in \mathbb{N}}$ of $A$ such that $F$ is differentiable on $\dot{E}$ and satisfies $\mathcal{N}\left(C_{i}, E_{i}\right)$ for all $i \in \mathbb{N}, \mathcal{N}\left(C^{*}, \dot{E}\right)$, and $\mathcal{N}\left(C^{*}, E_{i}\right)$ if $C_{i} \in \dot{\Gamma}$.

Remark 3.3. Observe that for set functions on $A$ the statements analogous to Remark 3.2 also hold true. 
4. Three basic theorems for the $\nu$-integral. In this section we prove three theorems which should be true in every reasonable theory of integration.

Theorem I shows that any linear combination of two $\nu$-integrals is itself a $\nu$-integral while Theorem II establishes a relation between $\nu$-integrals and $\nu$ integrals on $A$. Theorem III shows that a $\nu$-integral $F$ is uniquely determined by its derivative $\dot{F}$.

Theorem I (Linearity). Let $F_{1}$ and $F_{2}$ be two $\nu$-integrals and let $c_{1}$ and $c_{2}$ be real numbers. Then $c_{1} F_{1}+c_{2} F_{2}$ is a $\nu$-integral.

Pr o of. Denote by $\dot{E}_{j},\left(E_{i j}, C_{i j}\right)_{i \in \mathbb{N}}$ a division of $\mathbb{R}^{n}$ which corresponds to $F_{j}(j=1,2)$. Then we form a new division of $\mathbb{R}^{n}$ by taking $\dot{E}_{1} \cap \dot{E}_{2}$, $\left(\dot{E}_{1} \cap E_{i 2}, C_{i 2}\right)_{i \in \mathbb{N}},\left(\dot{E}_{2} \cap E_{i 1}, C_{i 1}\right)_{i \in \mathbb{N}},\left(E_{i 1} \cap E_{j 2}, \widetilde{C}_{i j}\right)_{i, j \in \mathbb{N}}$, where

$$
\widetilde{C}_{i j}= \begin{cases}C_{j 2} & \text { if } C_{i 1} \in \dot{\Gamma} \text { and } C_{j 2} \in \Gamma, \\ C_{i 1} & \text { if } C_{i 1} \in \Gamma \text { and } C_{j 2} \in \dot{\Gamma}, \\ C_{i 1} \text { resp. } C_{j 2} & \text { in all other cases in which } C_{i 1} \succeq C_{j 2} \text { resp. } C_{j 2} \succeq C_{i 1} .\end{cases}
$$

(If there are two possibilities in the last case, the choice is arbitrary.)

Obviously $F=c_{1} F_{1}+c_{2} F_{2}$ is differentiable on $\dot{E}_{1} \cap \dot{E}_{2}$ and by Remark 3.2, $F$ satisfies $\mathcal{N}\left(C^{*}, \dot{E}_{1} \cap \dot{E}_{2}\right)$.

Next consider $\left(\dot{E}_{1} \cap E_{i 2}, C_{i 2}\right)(i \in \mathbb{N})$. Assuming first that $C_{i 2} \in \Gamma$ we know that $C_{i 2} \succeq C^{*}$ and since $F_{1}$ satisfies $\mathcal{N}\left(C^{*}, \dot{E}_{1} \cap E_{i 2}\right)$ it also satisfies $\mathcal{N}\left(C_{i 2}, \dot{E}_{1} \cap E_{i 2}\right)$ by Remark 3.2. Since the latter is also true for $F_{2}$ we conclude that it must be true for $F$. On the other hand, suppose $C_{i 2} \in$ $\dot{\Gamma}$. Since $F_{1}$ is differentiable on the null set $\dot{E}_{1} \cap E_{i 2}$ we know again by Remark 3.2 that $F_{1}$ satisfies $\mathcal{N}\left(C_{i 2}, \dot{E}_{1} \cap E_{i 2}\right)$ and thus the same holds for $F$. In addition, both $F_{1}$ and $F_{2}$ satisfy $\mathcal{N}\left(C^{*}, \dot{E}_{1} \cap E_{i 2}\right)$ and therefore the same is true for $F$.

Analogously one discusses the pairs $\left(\dot{E}_{2} \cap E_{i 1}, C_{i 1}\right)$ for $i \in \mathbb{N}$.

It remains to examine $\left(E_{i 1} \cap E_{j 2}, \widetilde{C}_{i j}\right)$ for $i, j \in \mathbb{N}$. If $C_{i 1} \in \dot{\Gamma}$ and $C_{j 2} \in \Gamma$ then $F_{1}$ satisfies $\mathcal{N}\left(C^{*}, E_{i 1} \cap E_{j 2}\right)$ since $C_{i 1} \in \dot{\Gamma}$ and thus $F_{1}$ satisfies $\mathcal{N}\left(C_{j 2}, E_{i 1} \cap E_{j 2}\right)$ and the same holds for $F$. Analogously $F$ satisfies $\mathcal{N}\left(\widetilde{C}_{i j}, E_{i 1} \cap E_{j 2}\right)$ if $C_{i 1} \in \Gamma$ and $C_{j 2} \in \dot{\Gamma}$. The remaining cases are obvious since both $\Gamma$ and $\dot{\Gamma}$ are ordered, and thus $F$ is indeed a $\nu$-integral.

If $A \in \mathcal{B}$ and $F: \mathcal{B} \rightarrow \mathbb{R}$ is a set function we denote by $\left.F\right|_{A}$ the restriction of $F$ to $\mathcal{B}(A)$.

Theorem II (Restriction and Extension). Let $A \in \mathcal{B}$ and $F: \mathcal{B}(A) \rightarrow \mathbb{R}$.

(1) If there exists a $\nu$-integral $\widetilde{F}: \mathcal{B} \rightarrow \mathbb{R}$ with $\left.\widetilde{F}\right|_{A}=F$ then $F$ is a $\nu$-integral on $A$. 
(2) If $F$ is a $\nu$-integral on $A$ then the set function $\widetilde{F}: \mathcal{B} \rightarrow \mathbb{R}$ defined by $\widetilde{F}(X)=F(A \cap X)(X \in \mathcal{B})$ is a $\nu$-integral with $\left.\widetilde{F}\right|_{A}=F$.

Proof. (1) Denote by $\dot{E},\left(E_{i}, C_{i}\right)_{i \in \mathbb{N}}$ a division of $\mathbb{R}^{n}$ which corresponds to $\widetilde{F}$. Obviously $\dot{E} \cap A^{\circ},\left(\dot{E} \cap \partial A, C^{*}\right),\left(E_{i} \cap A, C_{i}\right)_{i \in \mathbb{N}}$ is a suitable division of $A$ for $F$.

(2) $\widetilde{F}$ is additive, $\dot{\widetilde{F}}=0$ on $A^{\text {c }}$, and denote by $\dot{E},\left(E_{i}, C_{i}\right)_{i \in \mathbb{N}}$ a division of $A$ corresponding to $F$. Then a division of $\mathbb{R}^{n}$ is given by

$$
\dot{E} \cup A^{\mathrm{c}}, \quad\left(E_{i} \cap A^{\circ}, C_{i}\right)_{C_{i} \in \dot{\Gamma}}, \quad\left(E_{i} \cap \partial A, C^{*}\right)_{C_{i} \in \dot{\Gamma}}, \quad\left(E_{i}, C_{i}\right)_{C_{i} \in \Gamma} .
$$

Since $\dot{E}, E_{i} \cap A^{\circ} \subseteq A^{\circ}$ we see immediately that $\widetilde{F}$ is differentiable on $\dot{E}$ (with $\dot{\widetilde{F}}=\dot{F})$ and satisfies $\mathcal{N}\left(C^{*}, \dot{E} \cup A^{\mathrm{c}}\right)$ as well as $\mathcal{N}\left(C_{i}, E_{i} \cap A^{\circ}\right)$ and $\mathcal{N}\left(C^{*}, E_{i} \cap A^{\circ}\right)$ for all $i \in \mathbb{N}$ with $C_{i} \in \dot{\Gamma}$.

Let us prove that $\widetilde{F}$ satisfies $\mathcal{N}\left(C_{i}, E_{i}\right)$ for $C_{i} \in \Gamma$. For given $\varepsilon, K>0$ find $\widetilde{K}>0$ by Axiom 2 and then choose $\widetilde{\Delta}>0$ and $\widetilde{\delta}: E_{i} \rightarrow \mathbb{R}^{+}$for $\varepsilon$ and $\widetilde{K}$ witnessing that $F$ satisfies $\mathcal{N}\left(C_{i}, E_{i}\right)$. Again Axiom 2 gives $\Delta>0$ and $\delta^{*}$ : $E_{i} \rightarrow \mathbb{R}^{+}$. Set $\delta=\min \left(\widetilde{\delta}, \delta^{*}\right)$ and let $\left\{\left(x_{k}, A_{k}\right)\right\}$ be an $\left(E_{i}, \delta\right)$-fine sequence with $\left\{A_{k}\right\} \in C_{i}(K, \Delta)$. Then $\left\{A \cap A_{k}\right\} \in C_{i}(\widetilde{K}, \widetilde{\Delta})$ by Axiom 2 and thus

$$
\sum\left|\widetilde{F}\left(A_{k}\right)\right|=\sum\left|F\left(A \cap A_{k}\right)\right| \leq \varepsilon .
$$

The proof that $\widetilde{F}$ satisfies $\mathcal{N}\left(C^{*}, E_{i} \cap \partial A\right)$ if $C_{i} \in \dot{\Gamma}$ is the same (since $F$ satisfies $\left.\mathcal{N}\left(C^{*}, E_{i}\right)\right)$, and therefore $\widetilde{F}$ is indeed a $\nu$-integral.

Theorem III (Positivity and Uniqueness). (1) Let $A \in \mathcal{B}$ and $F$ : $\mathcal{B}(A) \rightarrow \mathbb{R}$ be a $\nu$-integral on $A$ with $\dot{F} \geq 0$ a.e. (on $\left.A^{\circ}\right)$. Then $F(A) \geq 0$.

(2) If $F: \mathcal{B} \rightarrow \mathbb{R}$ is a $\nu$-integral, $A \in \mathcal{B}$ and $\dot{F} \geq 0$ a.e. on $A$, then $F(A) \geq 0$.

(3) Assume $F_{1}$ and $F_{2}$ to be two $\nu$-integrals with $\dot{F}_{1}=\dot{F}_{2}$ a.e. Then $F_{1}(B)=F_{2}(B)$ for every $B \in \mathcal{B}$.

Proof. Of course (1) implies (2) and (3) by Theorem II and so let us prove (1).

Assume $\dot{E},\left(E_{i}, C_{i}\right)_{i \in \mathbb{N}}$ to be a division of $A$ which corresponds to $F$, denote by $E$ a null set with $\partial A \subseteq E \subseteq A$ and $\dot{F} \geq 0$ on $A-E$, and let $K^{*}$ and $K_{i}^{*}$ be the numbers from the Decomposition Lemma.

Let $\varepsilon>0$. If $x \in \dot{E}$ there is a $\delta(x)>0$ with $\delta(x) \leq \operatorname{dist}\left(x, \mathbb{R}^{n}-A^{\circ}\right)$ such that $\left.\left.|F(B)-\dot{F}(x)| B\right|_{n}|\leq \varepsilon| B\right|_{n} /\left(1+|A|_{n}\right)$ for every $B \in \mathcal{D}\left(K^{*}\right)$ with $x \in B$ and $d(B)<\delta(x)$.

Since $|E \cap \dot{E}|_{n}=0$ we may also assume by Remark 3.2(iii) that $\sum\left|F\left(A_{k}\right)\right|$ $\leq \varepsilon$ for any $(E \cap \dot{E}, \delta)$-fine sequence $\left\{\left(x_{k}, A_{k}\right)\right\}$ with $A_{k} \in \mathcal{D}\left(K^{*}\right)$ for all $k$.

Furthermore, for $\varepsilon / 2^{i}$ and $K_{i}^{*}$ we find $\Delta_{i}>0$ and $\delta: E_{i} \rightarrow \mathbb{R}^{+}$corresponding to both null conditions, $\mathcal{N}\left(C_{i}, E_{i}\right)(i \in \mathbb{N})$ and $\mathcal{N}\left(C^{*}, E_{i} \cap \partial A\right)$, in case $C_{i} \in \dot{\Gamma}(i \in \mathbb{N})$, which are satisfied by $F$. 
We have taken the same $\delta$-function in all previous situations, since we may make it smaller finitely many times.

Thus by the Decomposition Lemma there is a $\delta$-fine partition $\left\{\left(x_{k}, A_{k}\right)\right\}$ of $A$ such that

- $\sum_{x_{k} \in \dot{E}-E} F\left(A_{k}\right) \geq \sum_{x_{k} \in \dot{E}-E} \dot{F}\left(x_{k}\right)\left|A_{k}\right|_{n}-\sum_{x_{k} \in \dot{E}-E} \frac{\varepsilon\left|A_{k}\right|_{n}}{1+|A|_{n}} \geq-\varepsilon$,

- $\sum_{x_{k} \in \dot{E} \cap E}\left|F\left(A_{k}\right)\right| \leq \varepsilon$,

- if $C_{i} \in \Gamma$ then $\sum_{x_{k} \in E_{i}}\left|F\left(A_{k}\right)\right| \leq \varepsilon / 2^{i}$,

- if $C_{i} \in \dot{\Gamma}$ then $\sum_{x_{k} \in E_{i} \cap A^{\circ}}\left|F\left(A_{k}\right)\right| \leq \varepsilon / 2^{i}$ and $\sum_{x_{k} \in E_{i} \cap \partial A}\left|F\left(A_{k}\right)\right| \leq \varepsilon / 2^{i}$, and so by additivity $F(A) \geq-5 \varepsilon$. Since $\varepsilon$ was arbitrary we conclude that $F(A) \geq 0$.

5. Integrable functions and their properties. Here we consider integrability of point functions, and we summarize the basic properties of the associated integral which follow from Theorems I-III. Furthermore, we show that our integration process generalizes the one of Lebesgue. Finally, the Fundamental Theorem characterizes those set functions which allow an integral representation.

5(a). $\nu$-integrable functions. If $A \subseteq \mathbb{R}^{n}$ and $f$ is a real-valued function defined at least on $A$ then we define $f_{A}: \mathbb{R}^{n} \rightarrow \mathbb{R}$ by

$$
f_{A}(x)= \begin{cases}f(x) & \text { if } x \in A \\ 0 & \text { otherwise. }\end{cases}
$$

For any $f: \mathbb{R}^{n} \rightarrow \mathbb{R}$ we denote by $\operatorname{supp} f$ as usual the closure of the set of points where $f$ is different from zero.

We call $f: \mathbb{R}^{n} \rightarrow \mathbb{R}$ with compact support $\nu$-integrable if there exists a $\nu$-integral $F: \mathcal{B} \rightarrow \mathbb{R}$ with $\dot{F}=f$ a.e., and we write

$$
\int f=F\left(\mathbb{R}^{n}\right) \quad \text { (see Thm. III and Remark 3.1). }
$$

Here Theorem III implies that $F$ is unique and has compact support. We denote by $\mathcal{I}_{\nu}$ the class of all $\nu$-integrable functions.

Let $A$ be a measurable subset of $\mathbb{R}^{n}$ and $f$ be a real-valued function which is defined at least on $A$. Then we call $f \nu$-integrable on $A$ if $f_{A}$ belongs to $\mathcal{I}_{\nu}$, and in this case we write

$$
\int_{A}^{\nu} f={ }^{\nu} f_{A} .
$$

We denote by $\mathcal{I}_{\nu}(A)$ the class of all functions which are $\nu$-integrable on $A$. 
Re m ark 5.1. (i) By the second part of the definition we have $f \in \mathcal{I}_{\nu}\left(\mathbb{R}^{n}\right)$ iff $f \in \mathcal{I}_{\nu}$, and ${ }^{\nu} \int_{\mathbb{R}^{n}} f={ }^{\nu} \int f$.

(ii) If there is no danger of misunderstanding we will often omit the superscript $\nu$ and write $\int f$ resp. $\int_{A} f$ instead of $\int^{\nu} f$ resp. ${ }^{\nu} \int_{A} f$.

(iii) For $A \in \mathcal{B}$ Theorem II implies that $f \in \mathcal{I}_{\nu}(A)$ iff there exists a $\nu$-integral $F$ on $A$ with $\dot{F}=f$ a.e. on $A$, and in that case $F(A)={ }^{\nu} \int_{A} f$.

(iv) By Banach's theorem [Saks, p. 112] every $f \in \mathcal{I}_{\nu}$ is measurable since the "ordinary" derivative of $F$ exists a.e.

Proposition 5.1. (i) Let $f \in \mathcal{I}_{\nu}$ and denote by $F$ the $\nu$-integral with $\dot{F}=f$ a.e. Then $f \in \mathcal{I}_{\nu}(A)$ and $\int_{A} f=F(A)$ for all $A \in \mathcal{B}$.

(ii) If $f_{1}$ and $f_{2}$ belong to $\mathcal{I}_{\nu}$ and $c_{1}, c_{2} \in \mathbb{R}$ then $c_{1} f_{1}+c_{2} f_{2} \in \mathcal{I}_{\nu}$ and $\int\left(c_{1} f_{1}+c_{2} f_{2}\right)=c_{1} \int f_{1}+c_{2} \int f_{2}$.

(iii) Assume $f \in \mathcal{I}_{\nu}$ with $f \geq 0$ a.e. Then $\int f \geq 0$.

(iv) Any function $f: \mathbb{R}^{n} \rightarrow \mathbb{R}$ with compact support and $f=0$ a.e. is $\nu$-integrable with $\int f=0$.

Proof. (i) Let $A \in \mathcal{B}$. Then by Theorem II the set function $G: \mathcal{B} \rightarrow \mathbb{R}$ defined by $G(X)=F(A \cap X)(X \in \mathcal{B})$ is a $\nu$-integral with $\dot{G}=f_{A}$ a.e. Thus $f \in \mathcal{I}_{\nu}(A)$ and $\int_{A} f=G(I)=F(A)$ where $I$ denotes an interval of support of $G$ containing $A$.

(ii) See Theorem I.

(iii) By Theorem III.

(iv) Clearly, $F: \mathcal{B} \rightarrow \mathbb{R}$ defined by $F(B)=0$ for all $B \in \mathcal{B}$ is a $\nu$-integral.

Remark 5.2. (i) If $f \in \mathcal{I}_{\nu}$ then by the above proposition all characteristic functions $\chi_{A}$ with $A \in \mathcal{B}$ are multipliers for $f$, i.e. $f \chi_{A} \in \mathcal{I}_{\nu}$.

(ii) By part (iv) of the above proposition we can and will consider $\nu$ integrability also for functions which are only defined a.e.

Proposition 5.2 gives the full additivity of our integral.

Proposition 5.2. Let $A \in \mathcal{B}, f: A \rightarrow \mathbb{R}$ and assume $A$ to be the finite union of sets $A_{k} \in \mathcal{B}$ with disjoint interiors. Then $f \in \mathcal{I}_{\nu}(A)$ iff $f \in \mathcal{I}_{\nu}\left(A_{k}\right)$ for all $k$, and in that case

$$
\int_{A} f=\sum \int_{A_{k}} f
$$

Proof. If $f \in \mathcal{I}_{\nu}(A)$ then $f_{A_{k}}=\left(f_{A}\right)_{A_{k}} \in \mathcal{I}_{\nu}$ by Proposition 5.1. Conversely, suppose $f \in \mathcal{I}_{\nu}\left(A_{k}\right)$ for all $k$. Then $\sum f_{A_{k}} \in \mathcal{I}_{\nu}$ with $\int \sum f_{A_{k}}=$ $\sum \int f_{A_{k}}=\sum \int_{A_{k}} f$, and since $f_{A}=\sum f_{A_{k}}$ a.e., by Proposition 5.1(iv) we get $f_{A} \in \mathcal{I}_{\nu}$ with $\int_{A} f=\int \sum f_{A_{k}}$.

5(b). Relation to Lebesgue integration. Next we prove that for nonnegative functions our process of integration coincides with Lebesgue's. 
For a measurable subset $A$ of $\mathbb{R}^{n}$ we denote by $\mathcal{L}(A)$ the set of all measurable functions $f: A \rightarrow \mathbb{R}$ for which a finite Lebesgue integral $\int_{A}|f|$ exists.

Theorem IV (Extension of Lebesgue Integration). Let $f: \mathbb{R}^{n} \rightarrow \mathbb{R}$ be non-negative with compact support. Then $f \in \mathcal{I}_{\nu}$ iff $f \in \mathcal{L}\left(\mathbb{R}^{n}\right)$, and in that case

$$
\int_{\mathbb{R}^{n}}^{\nu} f=\int_{\mathbb{R}^{n}}^{\mathcal{L}} f .
$$

Pro of. We first assume $f \in \mathcal{L}\left(\mathbb{R}^{n}\right)$ and define an additive set function $F: \mathcal{B} \rightarrow \mathbb{R}$ by $F(B)={ }^{\mathcal{L}} \int_{B} f(B \in \mathcal{B})$. By Lebesgue's theorem [Saks, p. 118] $F$ is "derivable in the general sense" with $\dot{F}=f$ on a set $\dot{E}$ with $\left|\mathbb{R}^{n}-\dot{E}\right|_{n}=0$. By $\left(\dot{\Gamma}_{2}\right)$ of Section 1 there is a control condition $C \in \dot{\Gamma}$ with $\mathbb{R}^{n}-\dot{E} \in \mathcal{E}(C)$ and thus $\dot{E},\left(\mathbb{R}^{n}-\dot{E}, C\right)$ is a division of $\mathbb{R}^{n}$.

We will show that $F$ satisfies $\mathcal{N}\left(C^{*}, \mathbb{R}^{n}\right)$ and $\mathcal{N}\left(C, \mathbb{R}^{n}-\dot{E}\right)$. Then $F$ is a $\nu$-integral with $\dot{F}=f$ a.e., hence $f \in \mathcal{I}_{\nu}$ and if $I$ denotes an interval of support of $F$ containing supp $f$ we get

$$
\int_{\mathbb{R}^{n}}^{\nu} f=F(I)=\int_{\mathbb{R}^{n}}^{\mathcal{L}} f .
$$

- F satisfies $\mathcal{N}\left(C^{*}, \mathbb{R}^{n}\right)$. Indeed, let $\varepsilon, K>0$. By the absolute continuity of the Lebesgue integral there is an $\eta>0$ such that ${ }^{\mathcal{L}} \int_{B} f<\varepsilon$ for any measurable $B$ with $|B|_{n}<\eta$.

By the assumption $\left(\Gamma_{2}\right)$ on $C^{*}$ there are a $\Delta>0$ and $\delta: \mathbb{R}^{n} \rightarrow \mathbb{R}^{+}$such that $\left|\bigcup A_{k}\right|_{n}<\eta$ for every $\left(\mathbb{R}^{n}, \delta\right)$-fine sequence $\left\{\left(x_{k}, A_{k}\right)\right\}$ with $\left\{A_{k}\right\} \in$ $C^{*}(K, \Delta)$. Consequently, for each such sequence,

$$
\sum\left|F\left(A_{k}\right)\right|=\int_{\cup A_{k}}^{\mathcal{L}} f<\varepsilon .
$$

- F satisfies $\mathcal{N}\left(C, \mathbb{R}^{n}-\dot{E}\right)$. Indeed, for $\varepsilon, K>0$ let $\eta>0$ be as before and find an open set $G$ containing $\mathbb{R}^{n}-\dot{E}$ with $|G|_{n}<\eta$. If $x \in \mathbb{R}^{n}-\dot{E}$ we choose a $\delta(x)>0$ with $B(x, \delta(x)) \subseteq G$, and we set $\Delta=1$. Then for any $\left(\mathbb{R}^{n}-\dot{E}, \delta\right)$-fine sequence $\left\{\left(x_{k}, A_{k}\right)\right\}$ with $\left\{A_{k}\right\} \in C(K, \Delta)$ it follows that $\left|\bigcup A_{k}\right|_{n} \leq|G|_{n}<\eta$, and hence

$$
\sum\left|F\left(A_{k}\right)\right|=\int_{\cup A_{k}}^{\mathcal{L}} f<\varepsilon
$$

as before.

Conversely, suppose $f \in \mathcal{I}_{\nu}$. By Remark 5.1, $f$ is measurable, and the sequence $f_{k}=\min (f, k)$ is non-decreasing and converges to $f$. Since $\operatorname{supp} f_{k} \subseteq \operatorname{supp} f$, all $f_{k}$ belong to $\mathcal{L}\left(\mathbb{R}^{n}\right)$, and by the first part of the proof 
and the monotonicity of our integral we conclude that

$$
\int_{\mathbb{R}^{n}}^{\mathcal{L}} f=\lim \int_{\mathbb{R}^{n}}^{\mathcal{L}} f_{k}=\lim \int_{\mathbb{R}^{n}}^{\nu} f_{k} \leq \int_{\mathbb{R}^{n}} f<\infty,
$$

and therefore $f \in \mathcal{L}\left(\mathbb{R}^{n}\right)$.

The following corollary contains the monotone and dominated convergence theorems as well as Fatou's lemma, which are reduced by Theorem IV to the classical situation.

Corollary 5.1. Let $A$ be a bounded measurable subset of $\mathbb{R}^{n}$ and let $f$, $g, h, f_{k}$ be real-valued functions a.e. on $A$ with $g, h, f_{k} \in \mathcal{I}_{\nu}(A)(k \in \mathbb{N})$.

(i) For non-negative $f$ we have $f \in \mathcal{I}_{\nu}(A)$ iff $f \in \mathcal{L}(A)$, and in that case ${ }^{\nu} \int_{A} f={ }^{\mathcal{L}} \int_{A} f$.

(ii) If $f_{k} \nearrow f$ pointwise and the (existing) $\lim ^{\nu} \int_{A} f_{k}$ is finite, then $f \in$ $\mathcal{I}_{\nu}(A)$ and ${ }^{\nu} \int_{A} f=\lim ^{\nu} \int_{A} f_{k}$.

(iii) Assume that $g \leq f_{k} \leq h$ and $f_{k} \rightarrow f$ pointwise a.e. on $A$. Then $f \in \mathcal{I}_{\nu}(A), \lim ^{\nu} \int_{A} f_{k}$ exists and ${ }^{\nu} \int_{A} f=\lim ^{\nu} \int_{A} f_{k}$.

(iv) If $f_{k} \geq g$ on $A$ and $\liminf { }^{\nu} \int_{A} f_{k}<\infty$ then $\liminf f_{k}$ is finite a.e. on $A$ and belongs to $\mathcal{I}_{\nu}(A)$. Furthermore, ${ }^{\nu} \int_{A} \liminf f_{k} \leq \liminf { }^{\nu} \int_{A} f_{k}$.

5(c). The Fundamental Theorem. We call $F: \mathcal{B} \rightarrow \mathbb{R}$ an indefinite $\nu$-integral if there exists an $f \in \mathcal{I}_{\nu}$ with $F(B)={ }^{\nu} \int_{B} f$ for all $B \in \mathcal{B}$.

Analogously if $A \in \mathcal{B}$ then $F: \mathcal{B}(A) \rightarrow \mathbb{R}$ is said to be an indefinite $\nu$-integral on $A$ if there is an $f \in \mathcal{I}_{\nu}(A)$ such that $F(B)={ }^{\nu} \int_{B} f$ for all $B \in \mathcal{B}(A)$.

The next theorem characterizes the indefinite $\nu$-integrals as $\nu$-integrals.

Theorem V (Fundamental Theorem). (1) $F: \mathcal{B} \rightarrow \mathbb{R}$ is an indefinite $\nu$-integral iff it is a $\nu$-integral, and in that case $\dot{F}$ is $\nu$-integrable and $F(B)=$ ${ }^{\nu} \int_{B} \dot{F}$ for all $B \in \mathcal{B}$.

(2) Let $A \in \mathcal{B}$. Then $F: \mathcal{B}(A) \rightarrow \mathbb{R}$ is an indefinite $\nu$-integral on $A$ iff it is a $\nu$-integral on $A$, and in that case $\dot{F} \in \mathcal{I}_{\nu}(A)$ and $F(B)={ }^{\nu} \int_{B} \dot{F}$ for all $B \in \mathcal{B}(A)$.

Proof. (1) Assume $F$ to be an indefinite $\nu$-integral; thus there is an $f \in \mathcal{I}_{\nu}$ with $F(B)=\int_{B} f$ for all $B \in \mathcal{B}$ by definition. Since $f \in \mathcal{I}_{\nu}$ there exists a $\nu$-integral $G$ with $\dot{G}=f$ a.e. and, by Proposition 5.1(i), $G(B)=\int_{B} f$ for $B \in \mathcal{B}$ so that $F=G$. Further, since $\dot{F}=f$ a.e. we conclude that $\dot{F} \in \mathcal{I}_{\nu}$, and consequently $F(B)=\int_{B} \dot{F}$ for all $B \in \mathcal{B}$.

Now let $F$ be a $\nu$-integral. Then there exists an interval $I$ with $\dot{F}=0$ a.e. on $I^{\mathrm{c}}$, and we define $f: \mathbb{R}^{n} \rightarrow \mathbb{R}$ by $f(x)=\dot{F}(x)$ for all $x \in I$ where $\dot{F}(x)$ exists, and $f(x)=0$ elsewhere. Then $f$ has compact support, $\dot{F}=f$ a.e., and therefore $f \in \mathcal{I}_{\nu}$ with $F(B)=\int_{B} f$ for $B \in \mathcal{B}$ again by Proposition 5.1(i). 
(2) Assume first $f \in \mathcal{I}_{\nu}(A)$ with $F(B)=\int_{B} f(B \in \mathcal{B}(A))$, and let $G: \mathcal{B} \rightarrow \mathbb{R}$ be the $\nu$-integral with $\dot{G}=f_{A}$ a.e. Then $G(B)=\int_{B} f_{A}$ for $B \in \mathcal{B}$, and therefore $F=\left.G\right|_{A}$ is a $\nu$-integral on $A$ by Theorem II.

Conversely, if $F$ is a $\nu$-integral on $A$ then $G(X)=F(A \cap X)(X \in \mathcal{B})$ defines a $\nu$-integral (Thm. II), and therefore by (1) we have $G(X)=\int_{X} \dot{G}$ for $X \in \mathcal{B}$. Since $\dot{F}=\dot{G}$ a.e. on $A$ we conclude that $\dot{F} \in \mathcal{I}_{\nu}(A)$ and $F(B)=\int_{B} \dot{F}$ for $B \in \mathcal{B}(A)$.

Discussion. Let $F: \mathcal{B} \rightarrow \mathbb{R}$ be an additive set function and let $D$ be the set of points $x$ where $\dot{F}(x)$ exists. We assume that $D^{\mathrm{c}}$ is a null set and that $\dot{F}=0$ a.e. on $I^{\text {c }}$ for some interval $I$.

Usually the Fundamental Theorem is concerned with additional conditions on $F$ such that

$$
\dot{F} \in \mathcal{I}_{\nu}(B) \quad \text { and } \quad F(B)=\int_{B}^{\nu} \dot{F} \quad(\forall B \in \mathcal{B}) .
$$

Clearly, $\dot{F} \in \mathcal{I}_{\nu}(B)$ for all $B \in \mathcal{B}$ is equivalent to $\dot{F} \in \mathcal{I}_{\nu}$ (take $B=I$ ). Hence, by Theorem $\mathrm{V}(1),(*)$ means that $F$ is a $\nu$-integral. So, by recalling the definition of a $\nu$-integral (Section 3$)$, we see that in our case $(*)$ means that $F$ must satisfy the null conditions corresponding to some division of $\mathbb{R}^{n}$ with $\dot{E} \subseteq D$.

6. The Saks-Henstock Lemma. In this section we assume additionally that the control condition $C^{*}$ does not depend on $\Delta$, i.e. $C^{*}(K, \Delta)=$ $C^{*}(K)$. This restriction is, in particular, satisfied in our concrete theories and seems to be quite natural.

Now we can prove the Saks-Henstock Lemma which gives a characterization of integrable functions, half way between a descriptive definition (as ours) and Riemann-type definitions of the integral (which are discussed separately).

Lemma (Saks-Henstock). Let $A \in \mathcal{B}$. Then $f: A \rightarrow \mathbb{R}$ is $\nu$-integrable on $A$ iff there is an additive set function $F: \mathcal{B}(A) \rightarrow \mathbb{R}$ and a division $\dot{E}$, $\left(E_{i}, C_{i}\right)_{i \in \mathbb{N}}$ of $A$ with the following property:

$\forall \varepsilon>0, K>0, K_{i}>0(i \in \mathbb{N}) \exists \Delta_{i}>0(i \in \mathbb{N}), \delta: A \rightarrow \mathbb{R}^{+}$such that

$$
\left.\sum\left|F\left(A_{k}\right)-f\left(x_{k}\right)\right| A_{k}\right|_{n}\left|+\sum\right| F\left(A_{k}^{\prime}\right)-f\left(x_{k}^{\prime}\right)\left|A_{k}^{\prime}\right|_{n} \mid \leq \varepsilon
$$

for any $(A, \delta)$-fine sequence $\left\{\left(x_{k}, A_{k}\right)\right\} \cup\left\{\left(x_{k}^{\prime}, A_{k}^{\prime}\right)\right\}$ with $A_{k}, A_{k}^{\prime} \in \mathcal{B}(A)$ and

(i) if $x_{k} \in \dot{E}$ then $A_{k} \in \mathcal{D}(K)$, and $\left\{A_{k}: x_{k} \in E_{i}\right\} \in C_{i}\left(K_{i}, \Delta_{i}\right)$ $(i \in \mathbb{N})$,

(ii) $\left\{A_{k}^{\prime}\right\} \in C^{*}(K)$ and $x_{k}^{\prime} \in \dot{E} \cup \bigcup_{C_{i} \in \dot{\Gamma}} E_{i}$ for all $k$. 
Proof. Assume first that $f \in \mathcal{I}_{\nu}(A)$, denote by $F: \mathcal{B}(A) \rightarrow \mathbb{R}$ the $\nu$-integral on $A$ with $\dot{F}=f$ a.e., let $\dot{E},\left(E_{i}, C_{i}\right)_{i \in \mathbb{N}}$ be a division of $A$ corresponding to $F$, and let $\varepsilon, K, K_{i}>0$.

Since $\dot{F}=f$ a.e. there is a null set $\widetilde{E} \subseteq \dot{E}$ such that $\dot{F}=f$ on $\dot{E}-\widetilde{E}$ and for any $x \in \dot{E}-\widetilde{E}$ there exists a $\delta(x)>0$ with $B(x, \delta(x)) \subseteq A^{\circ}$ and $\left.\left.|F(B)-f(x)| B\right|_{n}|\leq \varepsilon| B\right|_{n} /\left(6\left(1+|A|_{n}\right)\right)$ for each $B \in \mathcal{D}(K)$ with $x \in B$ and $d(B)<\delta(x)$.

By Remark 1.1 we can find a $\delta: \widetilde{E} \cup(A-\dot{E}) \rightarrow \mathbb{R}^{+}$which implies $\sum\left|f\left(x_{k}\right)\right|\left|A_{k}\right|_{n} \leq \varepsilon / 6$ for any $(\widetilde{E} \cup(A-\dot{E}), \delta)$-fine sequence $\left\{\left(x_{k}, A_{k}\right)\right\}$, and by Remark 3.2 we may assume $\sum\left|F\left(A_{k}\right)\right| \leq \varepsilon / 6$ for each $(\widetilde{E}, \delta)$-fine sequence $\left\{\left(x_{k}, A_{k}\right)\right\}$ with all $A_{k}$ in $\mathcal{D}(K)$.

Furthermore, $F$ satisfies $\mathcal{N}\left(C_{i}, E_{i}\right)(i \in \mathbb{N})$ and $\mathcal{N}\left(C^{*}, A-\bigcup_{C_{i} \in \Gamma} E_{i}\right)$ (see the end of Remark 3.2(i)), which yields $\Delta_{i}>0$ and $\delta_{i}: E_{i} \rightarrow \mathbb{R}^{+}$for $\varepsilon /\left(6 \cdot 2^{i}\right)$ and $K_{i}$, and also $\widetilde{\delta}: A-\bigcup_{C_{i} \in \Gamma} E_{i} \rightarrow \mathbb{R}^{+}$for $\varepsilon / 6$ and $K$.

Finally, write $\dot{E}=\bigcup_{j \in \mathbb{N}} T_{j}$ with $T_{j}=\{x \in \dot{E}: j-1 \leq|f(x)|<j\}$ and take $\delta_{j}^{*}: T_{j} \rightarrow \mathbb{R}^{+}$such that $\left|\bigcup A_{k}\right|_{n} \leq \varepsilon /\left(6 j 2^{j}\right)$ for any $\left(T_{j}, \delta_{j}^{*}\right)$-fine sequence $\left\{\left(x_{k}, A_{k}\right)\right\}$ with $\left\{A_{k}\right\} \in C^{*}(K)$ by the assumption $\left(\Gamma_{2}\right)$ on $C^{*}$ (see Section 1).

We may assume $\delta \leq \delta_{i}(i \in \mathbb{N}), \delta \leq \widetilde{\delta}$ and $\delta \leq \delta_{j}^{*}(j \in \mathbb{N})$.

Now let $\left\{\left(x_{k}^{\prime}, A_{k}^{\prime}\right)\right\} \cup\left\{\left(x_{k}, A_{k}\right)\right\}$ be an $(A, \delta)$-fine sequence with $A_{k}^{\prime}, A_{k} \in$ $\mathcal{B}(A)$ satisfying (i) and (ii). Then

$$
\begin{aligned}
& \left.\sum\left|F\left(A_{k}^{\prime}\right)-f\left(x_{k}^{\prime}\right)\right| A_{k}^{\prime}\right|_{n}\left|+\sum\right| F\left(A_{k}\right)-f\left(x_{k}\right)\left|A_{k}\right|_{n} \mid \\
& \leq \sum_{j \in \mathbb{N}}\left|F\left(A_{k}^{\prime}\right)\right|+\sum_{x_{k}^{\prime} \in T_{j}}\left|f\left(x_{k}^{\prime}\right)\right|\left|A_{k}^{\prime}\right|_{n} \\
& \quad+\left[\sum_{x_{k}^{\prime} \notin \dot{E}}\left|f\left(x_{k}^{\prime}\right)\right|\left|A_{k}^{\prime}\right|_{n}+\sum_{x_{k} \in \tilde{E} \cup(A-\dot{E})}\left|f\left(x_{k}\right)\right|\left|A_{k}\right|_{n}\right] \\
& \quad+\left.\sum_{x_{k} \in \dot{E}-\tilde{E}}\left|F\left(A_{k}\right)-f\left(x_{k}\right)\right| A_{k}\right|_{n}\left|+\sum_{x_{k} \in \tilde{E}}\right| F\left(A_{k}\right)\left|+\sum_{i \in \mathbb{N}} \sum_{x_{k} \in E_{i}}\right| F\left(A_{k}\right) \mid \\
& \leq \frac{\varepsilon}{6}+\sum_{j \in \mathbb{N}} j\left|\bigcup_{x_{k}^{\prime} \in T_{j}} A_{k}^{\prime}\right|_{n}+\frac{\varepsilon}{6}+\frac{\varepsilon}{6\left(1+|A|_{n}\right)} \sum\left|A_{k}\right|_{n}+\frac{\varepsilon}{6}+\sum_{i \in \mathbb{N}} \frac{\varepsilon}{6 \cdot 2^{i}} \leq \varepsilon
\end{aligned}
$$

as desired.

Conversely, suppose $F: \mathcal{B}(A) \rightarrow \mathbb{R}$ is additive and $\dot{E},\left(E_{i}, C_{i}\right)_{i \in \mathbb{N}}$ is a division of $A$ with the Saks-Henstock property.

By using Vitali's covering theorem we first prove that $\dot{F}=f$ a.e. on $\dot{E}$ (cf. [Yee-Na]). Denote by $E$ the set of $x \in \dot{E}$ where $F$ is not differentiable or $\dot{F}(x) \neq f(x)$. Then for any $x \in E$ there are $\varepsilon(x), K(x)>0$ such that for every $\delta>0$ there exists a set $B \in \mathcal{D}(K(x)), B \in \mathcal{B}(A)$ with $x \in B$ and 
$d(B)<\delta$ but

$$
\left.\left.|F(B)-f(x)| B\right|_{n}|>\varepsilon(x)| B\right|_{n} .
$$

For $m, k \in \mathbb{N}$ set $E_{m, k}=\{x \in E: \varepsilon(x) \geq 1 / m, K(x) \leq k\}$ so that $E=\bigcup_{m, k \in \mathbb{N}} E_{m, k}$. It suffices to prove that $\left|E_{m, k}\right|_{n}=0$ for all $m, k \in \mathbb{N}$.

Fix $m, k \in \mathbb{N}$, let $\varepsilon>0$ and determine $\Delta_{i}>0$ and $\delta: A \rightarrow \mathbb{R}^{+}$by the Saks-Henstock property for $\varepsilon / m, K=k$ and $K_{i}=1(i \in \mathbb{N})$, where we may assume $\delta(x)<\operatorname{dist}\left(x, \mathbb{R}^{n}-A^{\circ}\right)$ if $x \in A^{\circ}$. We denote by $\mathcal{V}$ the set of all $B \in \mathcal{D}(k)$ for which there exists an $x \in B \cap E_{m, k}$ with $d(B)<\delta(x)$ and $\left.\left.|F(B)-f(x)| B\right|_{n}|>| B\right|_{n} / m$.

One easily verifies that $\mathcal{V}$ covers $E_{m, k}$ in the sense of Vitali, by the construction of $\varepsilon(x)$ and $K(x)$ above. Consequently (see, e.g., [Saks, p. 109]), we find at most countably many disjoint $A_{i} \in \mathcal{V}$ with $\left|E_{m, k}-\bigcup A_{i}\right|_{n}=0$ and, for each $A_{i}$, an $x_{i} \in A_{i} \cap E_{m, k}$ with $d\left(A_{i}\right)<\delta\left(x_{i}\right)$ and $\left.\left|F\left(A_{i}\right)-f\left(x_{i}\right)\right| A_{i}\right|_{n} \mid>$ $\left|A_{i}\right|_{n} / m$. Now using the Saks-Henstock property for $\left\{\left(x_{i}, A_{i}\right): 1 \leq i \leq l\right\}$ $(l \in \mathbb{N})$ we get

$$
\left|\bigcup_{i=1}^{l} A_{i}\right|_{n}=\sum_{i=1}^{l}\left|A_{i}\right|_{n} \leq\left. m \sum_{i=1}^{l}\left|F\left(A_{i}\right)-f\left(x_{i}\right)\right| A_{i}\right|_{n} \mid \leq \varepsilon,
$$

and therefore $\left|E_{m, k}\right|_{n} \leq \varepsilon$. Since $\varepsilon$ was arbitrary, $\left|E_{m, k}\right|_{n}=0$.

By the assumptions on $\dot{\Gamma}$ we can find a $C \in \dot{\Gamma}$ with $E \in \mathcal{E}(C)$ and thus $\dot{E}-E,(E, C),\left(E_{i}, C_{i}\right)_{i \in \mathbb{N}}$ is a division of $A$. Now it suffices to show that $F$ is a $\nu$-integral on $A$ with $\dot{F}=f$ a.e. on $A$. Here we use the Saks-Henstock (SH) property and Remark 1.1:

To see that $F$ satisfies $\mathcal{N}(C, E), \mathcal{N}\left(C_{i}, E_{i}\right)$ we apply the SH-property with corresponding parameters (e.g., in the first case, with the same $\varepsilon, K$, and all $K_{i}=1$ ), where we only need the (i) part.

To see that $F$ satisfies $\mathcal{N}\left(C^{*}, \dot{E}-E\right), \mathcal{N}\left(C^{*}, E\right)$, and $\mathcal{N}\left(C^{*}, E_{i}\right)$ if $C_{i} \in \dot{\Gamma}$, we use in a similar way the (ii) part of the SH-property. Here, Remark 1.1 does not apply to $\mathcal{N}\left(C^{*}, \dot{E}-E\right)$; instead we split $\dot{E}-E$ into countably many parts where $f$ is bounded (as in the first part of our proof) and use $\left(\Gamma_{2}\right)$.

Corollary 6.1. If $A \in \mathcal{B}$ and if $f: A \rightarrow \mathbb{R}$ is $\nu$-integrable on $A$ then there exists a real number $J$ and a division $\dot{E},\left(E_{i}, C_{i}\right)_{i \in \mathbb{N}}$ of $A$ with the following property:

$\forall \varepsilon>0, K>0, K_{i}>0 \exists \Delta_{i}>0, \delta: A \rightarrow \mathbb{R}^{+}$such that

$$
\left|J-\left(\sum f\left(x_{k}\right)\left|A_{k}\right|_{n}+\sum f\left(x_{k}^{\prime}\right)\left|A_{k}^{\prime}\right|_{n}\right)\right| \leq \varepsilon
$$

for any $\delta$-fine partition $\left\{\left(x_{k}, A_{k}\right)\right\} \cup\left\{\left(x_{k}^{\prime}, A_{k}^{\prime}\right)\right\}$ of A satisfying

(i) if $x_{k} \in \dot{E}$ then $A_{k} \in \mathcal{D}(K)$, and $\left\{A_{k}: x_{k} \in E_{i}\right\} \in C_{i}\left(K_{i}, \Delta_{i}\right)$ $(i \in \mathbb{N})$,

(ii) $\left\{A_{k}^{\prime}\right\} \in C^{*}(K)$ and $x_{k}^{\prime} \in \dot{E} \cup \bigcup_{C_{i} \in \dot{\Gamma}} E_{i}$ for all $k$. 
The corollary is an immediate consequence of the Saks-Henstock Lemma (with $J={ }^{\nu} \int_{A} f$ ), and thus for any integrable function the value of the integral can be obtained as the limit of certain Riemann sums, since by Remark 2.1 the desired partitions exist: If we take $K \geq K^{*}, K_{i} \geq K_{i}^{*}$ with the parameters from the Decomposition Lemma we see that the properties (i) respectively (ii) of that lemma correspond to (i) respectively (ii) of our corollary.

The existence of such partitions also implies that the number $J$ is uniquely determined.

One might hope that the above corollary even gives a characterization of integrability, but this seems to be unlikely without further restrictions on $\mathcal{B}, \mathcal{D}, \dot{\Gamma}$ and $\Gamma$. However, in our concrete theories this will be the case.

\section{The transformation axiom and the transformation formula.}

Let $A$ be a measurable subset of $\mathbb{R}^{n}$. Then $\phi: A \rightarrow \mathbb{R}^{n}$ is called a transformation map if it is one-to-one and if $\phi$ and $\phi^{-1}$ are Lipschitzian.

Remark 7.1. (i) By the Kirszbraun-McShane theorem [Kir], [McSh] any transformation map $\phi: A \rightarrow \mathbb{R}^{n}$ has a Lipschitzian extension to the whole space which is a.e. differentiable according to Rademacher (see, e.g., [Fed, p. 216]). Furthermore, for any measurable subset $E$ of $A$ we have $|\phi(E)|_{n}=\mathcal{L} \int_{E}\left|\operatorname{det} \phi^{\prime}\right|$ (see, e.g., [Fed, p. 243]), where $\operatorname{det} \phi^{\prime}$ denotes the determinant of the derivative $\phi^{\prime}$ at all points where the latter exists uniquely, and is zero elsewhere.

(ii) Recall that for any $A \in \mathcal{B}$ and any transformation map $\phi: A \rightarrow \mathbb{R}^{n}$ we have $\phi\left(A^{\circ}\right)=\phi(A)^{\circ}$ by the domain invariance theorem (see, e.g., [Rot]).

To prove the transformation formula within our theory we assume that the semi-ring $\mathcal{B}$, the differentiation classes and the control conditions are invariant with respect to the transformation map.

Axiom 3 (transformation). For each $A \in \mathcal{B}$ and each transformation map $\phi: A \rightarrow \mathbb{R}^{n}$ the following shall be true:

(i) $\phi(A) \in \mathcal{B}$.

(ii) For all $K>0$ there exists a $\widetilde{K}>0$ such that $\phi(E) \in \mathcal{D}(\widetilde{K})$ for any subset $E$ of $A$ with $E \in \mathcal{D}(K)$.

(iii) For every $C \in \Gamma \cup \dot{\Gamma}$ we have

(a) $\forall K>0 \exists \widetilde{K}>0 \forall \widetilde{\Delta}>0 \exists \Delta>0$ such that for any sequence $\left\{A_{k}\right\} \in C(K, \Delta)$ with $A_{k} \subseteq A$ we have $\left\{\phi\left(A_{k}\right)\right\} \in C(\widetilde{K}, \widetilde{\Delta})$.

(b) $\phi(E) \in \mathcal{E}(C)$ whenever $E \subseteq A$ with $E \in \mathcal{E}(C)$.

Theorem VI (Transformation Formula). Let $A \in \mathcal{B}, \phi: A \rightarrow \mathbb{R}^{n}$ be a transformation map and let $f: \phi(A) \rightarrow \mathbb{R}$. Then $f \in \mathcal{I}_{\nu}(\phi(A))$ if and only 
if $(f \circ \phi)\left|\operatorname{det} \phi^{\prime}\right| \in \mathcal{I}_{\nu}(A)$, and in that case

$$
\int_{\phi(A)}^{\nu} f=\int_{A}^{\nu}(f \circ \phi)\left|\operatorname{det} \phi^{\prime}\right| .
$$

Proof. Since $\phi^{-1}$ is again a transformation map it suffices to consider the case when $f$ is $\nu$-integrable on $\phi(A)$.

There are $c_{1}, c_{2}>0$ such that $c_{2}\|x-y\| \leq\|\phi(x)-\phi(y)\| \leq c_{1}\|x-y\|$ for all $x, y \in A$.

Denote by $F: \mathcal{B}(\phi(A)) \rightarrow \mathbb{R}$ the $\nu$-integral on $\phi(A)$ with $\dot{F}=f$ a.e. on $\phi(A)$, let $\dot{E},\left(E_{i}, C_{i}\right)_{i \in \mathbb{N}}$ be a corresponding division of $\phi(A)$, and define an additive function $G: \mathcal{B}(A) \rightarrow \mathbb{R}$ by $G(X)=F(\phi(X))(X \in \mathcal{B}(A))$.

We will show that $G$ is a $\nu$-integral on $A$ with $\dot{G}=(f \circ \phi)\left|\operatorname{det} \phi^{\prime}\right|$ a.e. on $A$, which implies $(f \circ \phi)\left|\operatorname{det} \phi^{\prime}\right| \in \mathcal{I}_{\nu}(A)$ and ${ }^{\nu} \int_{A}(f \circ \phi)\left|\operatorname{det} \phi^{\prime}\right|=G(A)=$ $F(\phi(A))=\int_{\phi(A)} f$ by Theorem $\mathrm{V}(2)$.

Set $H(X)={ }^{\mathcal{L}} \int_{X}\left|\operatorname{det} \phi^{\prime}\right|_{A}(X \in \mathcal{B})$. Then there is a null set $E \subseteq \mathbb{R}^{n}$ such that $\dot{H}=\left|\operatorname{det} \phi^{\prime}\right|_{A}$ on $E^{\text {c }}$. We now show that $G$ is differentiable for all $x \in \phi^{-1}(\dot{E})-E$ with $\dot{G}(x)=\dot{F}(\phi(x))\left|\operatorname{det} \phi^{\prime}(x)\right|$. Fix an $x \in \phi^{-1}(\dot{E})-E$ and let $\varepsilon, K>0$. Find a $\widetilde{K}>0$ by (ii) of Axiom 3. Then there are $\delta_{1}, \delta_{2}>0$ such that

and

$$
\left.|H(B)-| \operatorname{det} \phi^{\prime}(x)|| B\right|_{n} \mid \leq \frac{\varepsilon|B|_{n}}{2(1+|\dot{F}(\phi(x))|)}
$$

$$
\left.|F(C)-\dot{F}(\phi(x))| C\right|_{n} \mid \leq \frac{\varepsilon|C|_{n}}{2 c_{1}^{n}}
$$

for all $B \in \mathcal{D}(K)$ with $x \in B$ and $d(B)<\delta_{1}$, and respectively for all $C \in \mathcal{D}(\widetilde{K})$ with $C \subseteq \phi(A), \phi(x) \in C$ and $d(C)<\delta_{2}$.

Now set $\delta=\min \left(\delta_{1}, \delta_{2} / c_{1}\right.$, $\left.\operatorname{dist}\left(x, \mathbb{R}^{n}-A^{\circ}\right)\right)$ and let $B \in \mathcal{D}(K)$ with $x \in B$ and $d(B)<\delta$. Then $\phi(B) \in \mathcal{D}(\widetilde{K})$ by Axiom $3,|\phi(B)|_{n} \leq c_{1}^{n}|B|_{n}$ since $\phi$ is Lipschitzian, and therefore

$$
\begin{aligned}
& \left.|G(B)-\dot{F}(\phi(x))| \operatorname{det} \phi^{\prime}(x)|| B\right|_{n}|\leq| F(\phi(B))-\dot{F}(\phi(x))|\phi(B)|_{n} \mid \\
& \quad+\left.|\dot{F}(\phi(x))||| \phi(B)\right|_{n}-\left.\left|\operatorname{det} \phi^{\prime}(x)\right||B|_{n}\left|\leq \frac{\varepsilon|\phi(B)|_{n}}{2 c_{1}^{n}}+\frac{\varepsilon|B|_{n}}{2} \leq \varepsilon\right| B\right|_{n}
\end{aligned}
$$

by Remark 7.1.

Let $\widetilde{E}=\phi^{-1}(\dot{E}) \cap E$ and take $C \in \dot{\Gamma}$ with $\widetilde{E} \in \mathcal{E}(C)$. Since $|\phi(\widetilde{E})|_{n}=0$ and $F$ is differentiable on $\phi(\widetilde{E})$ we know by Remark 3.2(iii) that $F$ satisfies $\mathcal{N}(C, \phi(\widetilde{E}))$.

Now, $\phi^{-1}(\dot{E})-E,(\widetilde{E}, C),\left(\phi^{-1}\left(E_{i}\right), C_{i}\right)_{i \in \mathbb{N}}$ is a division of $A$, and we will show that $G$ satisfies the corresponding null conditions. It suffices to prove that $G$ satisfies $\mathcal{N}\left(C^{*}, \phi^{-1}(\dot{E})-E\right)$ since the other conditions are 
proved analogously. So let $\varepsilon, K>0$, find $\widetilde{K}$ by Axiom 3(iii) and find $\widetilde{\Delta}>0$ and $\widetilde{\delta}: \dot{E} \rightarrow \mathbb{R}^{+}$for $\varepsilon$ and $\widetilde{K}$ witnessing that $F$ satisfies $\mathcal{N}\left(C^{*}, \dot{E}\right)$. Again by axiom 3(iii) there is a $\Delta>0$ and we set $\delta(x)=\widetilde{\delta}(\phi(x)) / c_{1}$ for $x \in$ $\phi^{-1}(\dot{E})-E$. If then $\left\{\left(x_{k}, A_{k}\right)\right\}$ denotes a $\left(\phi^{-1}(\dot{E})-E, \delta\right)$-fine sequence with $A_{k} \subseteq A$ and $\left\{A_{k}\right\} \in C^{*}(K, \Delta)$ we have

$$
\sum\left|G\left(A_{k}\right)\right|=\sum\left|F\left(\phi\left(A_{k}\right)\right)\right| \leq \varepsilon
$$

since $\left\{\left(\phi\left(x_{k}\right), \phi\left(A_{k}\right)\right)\right\}$ is an $(\dot{E}, \widetilde{\delta})$-fine sequence with $\left\{\phi\left(A_{k}\right)\right\} \in C^{*}(\widetilde{K}, \widetilde{\Delta})$.

Thus $G$ is a $\nu$-integral on $A$ with $\dot{G}(x)=\dot{F}(\phi(x))\left|\operatorname{det} \phi^{\prime}(x)\right|$ a.e. on $A$ and since $\dot{F}=f$ a.e. on $\phi(A)$ the theorem is proved.

Discussion. Axiom 3 relates "all" $A, \phi$ to $\nu=(\mathcal{B}, \mathcal{D}, \dot{\Gamma}, \Gamma)$ although in Theorem VI only a simple pair $(A, \phi)$ is involved. It is clear that one can formulate a weaker axiom corresponding to such an individual situation. For reasons of simplicity we have avoided the complications connected with such a refinement.

\section{References}

[Fed] H. Federer, Geometric Measure Theory, Springer, New York, 1969.

[Jar-Ku 1] J. Jarník and J. Kurzweil, A non-absolutely convergent integral which admits $C^{1}$ - transformations, Časopis Pěst. Mat. 109 (1984), 157-167.

[Jar-Ku 2] -, - A non-absolutely convergent integral which admits transformation and can be used for integration on manifolds, Czechoslovak Math. J. 35 (110) (1985), 116-139.

[Jar-Ku 3] - - - A new and more powerful concept of the PU-integral, ibid. 38 (113) (1988), 8-48.

[Ju] W. B. Jurkat, The Divergence Theorem and Perron integration with exceptional sets, ibid. 43 (118) (1993), 27-45.

[Ju-Kn] W. B. Jurkat and R. W. Knizia, A characterization of multi-dimensional Perron integrals and the fundamental theorem, Canad. J. Math. 43 (1991), 526-539.

[Kir] M. D. Kirszbraun, Über die zusammenziehende und Lipschitzsche Transformationen, Fund. Math. 22 (1934), 77-108.

[Maw] J. Mawhin, Generalized multiple Perron integrals and the Green-Goursat theorem for differentiable vector fields, Czechoslovak Math. J. 31 (106) (1981), 614-632.

[McSh] E. J. McShane, Extension of range of functions, Bull. Amer. Math. Soc. 40 (1934), 837-842.

[No] D. J. F. Nonnenmacher, Theorie mehrdimensionaler Perron-Integrale mit Ausnahmemengen, PhD thesis, Univ. of Ulm, 1990.

[Pf 1] W. F. Pfeffer, The multidimensional fundamental theorem of calculus, J. Austral. Math. Soc. 43 (1987), 143-170.

[Pf 2] -, The Gauß-Green Theorem, Adv. in Math. 87 (1991), 93-147.

[Pf 3] - A descriptive definition of a variational integral and applications, Indiana Univ. Math. J. 40 (1991), 259-270. 
[Pf-Ya] W. F. Pfeffer and W.-C. Yang, A multidimensional variational integral and its extensions, Real Anal. Exchange 15 (1989-1990), 111-169.

[Rot] J. J. Rotman, An Introduction to Algebraic Topology, Graduate Texts in Math., Springer, 1988.

[Saks] S. Saks, Theory of the Integral, Dover, New York, 1964.

[Weir] A. J. Weir, General Integration and Measure, Vol. 2, Cambridge University Press, 1974.

[Yee-Na] L. P. Yee and W. Naak-In, A direct proof that Henstock and Denjoy integrals are equivalent, Bull. Malaysian Math. Soc (2) 5 (1982), 43-47.

ABTEILUNG FÜR MATHEMATIK II UND V

UNIVERSITÄT ULM

D-89069 ULM, GERMANY 